\title{
ACCURATE DETECTION OF MOVING TARGETS VIA RANDOM SENSOR ARRAYS AND KERDOCK CODES
}

\author{
THOMAS STROHMER AND HAICHAO WANG
}

\begin{abstract}
The detection and parameter estimation of moving targets is one of the most important tasks in radar. Arrays of randomly distributed antennas have been popular for this purpose for about half a century. Yet, surprisingly little rigorous mathematical theory exists for random arrays that addresses fundamental question such as how many targets can be recovered, at what resolution, at which noise level, and with which algorithm. In a different line of research in radar, mathematicians and engineers have invested significant effort into the design of radar transmission waveforms which satisfy various desirable properties. In this paper we bring these two seemingly unrelated areas together. Using tools from compressive sensing we derive a theoretical framework for the recovery of targets in the azimuth-range-Doppler domain via random antennas arrays. In one manifestation of our theory we use Kerdock codes as transmission waveforms and exploit some of their peculiar properties in our analysis. Our paper provides two main contributions: (i) We derive the first rigorous mathematical theory for the detection of moving targets using random sensor arrays. (ii) The transmitted waveforms satisfy a variety of properties that are very desirable and important from a practical viewpoint. Thus our approach does not just lead to useful theoretical insights, but is also of practical importance. Various extensions of our results are derived and numerical simulations confirming our theory are presented.
\end{abstract}

\section{INTRODUCTION}

The detection and parameter estimation of moving targets is one of the most important radar applications. The use of antenna arrays greatly improves our ability to perform this task. Antenna arrays make it possible to estimate not only the range and Doppler frequency, but also the azimuth of the target. Furthermore, using multiple antennas can significantly increase signal strength and thus in turn can greatly enhance accuracy and our ability to locate low contrast targets ("faint" or "weak" targets).

Therefore it does not come as a surprise that in recent years radar systems employing multiple antennas at the transmitter and the receiver (also referred to as MIMO radar, where MIMO stands for multiple-input multiple-output) have attracted enormous attention in the engineering and signal processing community. Despite the significant resources that have been devoted to MIMO radar, there exists fairly little rigorous mathematical theory for MIMO radar that addresses fundamental questions, such as how many targets can be detected at which azimuthrange-Doppler resolution and at what signal-to-noise ratio. Existing theory focuses

Key words and phrases. Sparsity, Radar, Compressive Sensing, Random Sensor Arrays, MIMO, Kerdock Codes. 
mainly on the detection of a single target [12, 26]. Only very recently, in the footsteps of compressive sensing, do we see the emergence of a rigorous mathematical theory for MIMO radar that addresses the more realistic and more interesting case of multiple targets [33. However, for the widely popular case of randomly spaced antennas ${ }^{1}$, the mathematical theory is still in its infancy.

In an independent and seemingly disparate line of research in radar, mathematicians and engineers have devoted substantial efforts to the design of radar transmission waveforms that satisfy a variety of desirable properties. The vast majority of this research has focused on single antenna radar systems, and it is a priori not clear if and how these waveforms can be utilized for MIMO radar. In this paper we bring together these two independent areas of research, MIMO radar with random antenna arrays and radar waveform design, by developing a rigorous mathematical framework for accurate target detection via random arrays, which at the same time utilizes some of the most attractive radar waveforms, such as Kerdock codes.

A radar system illuminates a region of interest in order to detect the location, velocity, and reflectivity of the objects (targets) in its field of view. We consider the following standard (narrowband) radar model [32. Suppose a target located at range $r$ is traveling with constant velocity $v$ and has reflection coefficient a. Suppose further just for the moment that we have only one target, one transmitter and one receiver (in which case we cannot detect direction). After transmitting signal $s(t)$, the receiver observes the reflected signal

$$
y(t)=a s\left(t-\tau_{r}\right) e^{2 \pi i \omega_{v} t}
$$

where $\tau_{r}=2 r / c$ is the round trip time of flight, $c$ is the speed of light, $\omega_{v} \approx-2 \omega_{0} v / c$ is the Doppler shift, and $\omega_{0}$ is the carrier frequency. The basic idea is that the rangevelocity information $(r, v)$ of the target can be inferred from the observed time delayDoppler shift $\left(\tau_{r}, \omega_{v}\right)$ of $s$ in (1). For only one target this can be done conveniently by correlating the received signal $y$ with time-frequency shifted versions of the transmitted signal. Since we are dealing with bandlimited signals, it suffices to consider discrete signals sampled at a properly chosen rate $\Delta_{t}$. It is therefore common practice to compute

$$
V(\tau, \omega):=\sum_{l} y\left(l \Delta_{t}\right) \overline{\mathbf{s}\left(l \Delta_{t}-\tau\right) e^{2 \pi i \omega l}}
$$

and then locate the largest value of $|V(\tau, \omega)|$ in order to detect the target in the range-Doppler domain.

In the presence of multiple targets more sophisticated methods are necessary. In order to resolve azimuth in addition to range and Doppler, we need to employ an array of antennas. We assume an array of $N_{T}$ transmit and $N_{R}$ receiver antennas that are co-located (also known as mono-static radar) as illustrated in Figure 1 A more detailed description of the setup is postponed to Section 2, The transmit antennas send simultaneously probing signals, which can differ from antenna to antenna and can be chosen to our specifications. It is convenient to divide the region of interest into range-azimuth-Doppler cells corresponding to distance, direction and velocity, respectively. Let $\mathbf{A}$ be a measurement matrix whose columns correspond to the signal recorded at each receive antenna from a single unit-strength scatterer

\footnotetext{
${ }^{1}$ In this paper we only consider the case of co-located transmitters and receivers, which is the most relevant situation in practice. We do not discuss the case of widely separated antennas [15].
} 


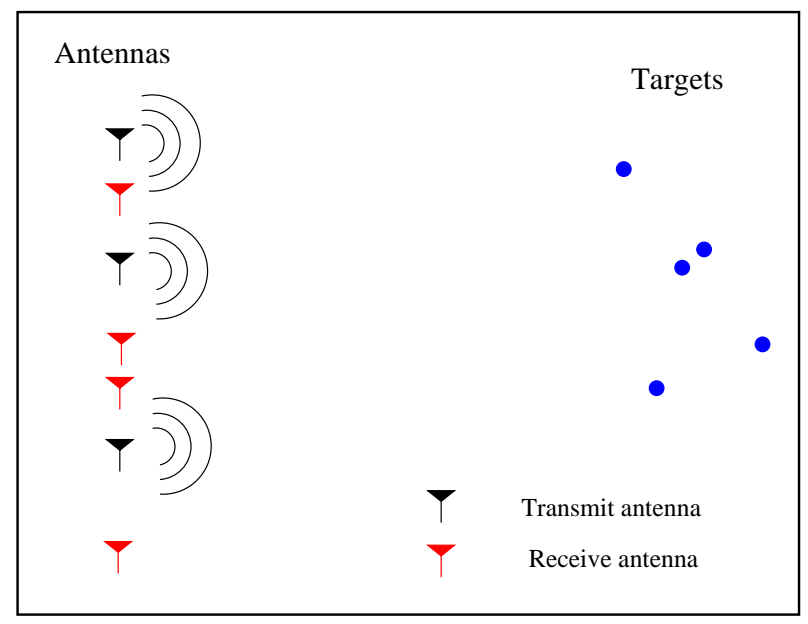

FiguRe 1.

at a specific range-azimuth-Doppler cell. Let $\mathbf{x}$ denote a vector whose elements represent the complex amplitudes of the scatterers. In many cases the radar scene is sparse in the sense that only a small fraction (often a very small fraction) of the cells is occupied by the objects of interest. In this case most of the entries of $\mathbf{x}$ will be zero, but we do not know which ones, otherwise we would have located the targets already. With $\mathbf{w}$ representing a noise vector, we are faced with the linear system of equations

$$
\mathbf{y}=\mathbf{A x}+\mathbf{w}
$$

where $\mathbf{y}$ is a vector of measurements collected by the receive antennas over an observation interval. Typically this system will be underdetermined, which implies that it will have infinitely many solutions. What comes to our rescue here is the sparsity of $\mathbf{x}$. While conventional radar processing techniques do not take full advantage of sparsity of the radar scene, the recent development of compressive sensing provides us with the possibility to optimally utilize this property [17, 31, 33]. The approach pursued in this paper to obtain a sparse solution of (3) is based on the lasso [36, which gained tremendous popularity in connection with compressive sensing. The lasso solves

$$
\min _{\mathbf{x}} \frac{1}{2}\|\mathbf{A x}-\mathbf{y}\|_{2}^{2}+\lambda\|\mathbf{x}\|_{1},
$$

where the parameter $\lambda>0$ trades off goodness of fit with sparsity.

However, one of the main challenges in bringing compressive sensing theory into radar is that in radar the sensing matrix $\mathbf{A}$ cannot be freely chosen. Its structure is dictated by the laws of physics on which radar is based. The crux is to carefully balance the desired resolution in the azimuth-range-Doppler domain with the degrees of freedom at our disposal in the formation of $\mathbf{A}$, such as the antenna locations and the transmit waveforms.

A great deal of work has been devoted in the mathematical and engineering literature to the design of radar transmission waveforms, see for instance [1, 3, 9, 13, 14, 29, 38 for a small sample of references. The design criteria for radar 
waveforms can be roughly split into two categories: (i) properties that are important from the viewpoint of hardware implementation, and (ii) properties that are relevant for target detection. Waveforms that fall in the first category are for example polyphase sequences, since they give rise to signals with low peak-to-average power ratid 3 (PAPR). A low PAPR is desirable in the digital-to-analog conversion of signals, since signals with large PAPR would require expensive power amplifiers. Polyphase sequences also have the advantage that they can be very convientiently implemented in hardware via simple look-up tables. The second category usually includes waveforms with low auto-correlation and (nearly) ideal ambiguity function. Quite a number of polyphase sequences, such as Alltop sequences or Kerdock codes, fall in this category. With the exception of [17] a rigorous mathematical theory concerning the benefits (or even optimality) of such sequences has only existed for the detection of a single target. Common to all these carefully constructed sequences in both categories is that they have been designed for single-antenna radar systems and it is a priori not clear at all if any of these sequences are useful in exploiting the potential benefits of a MIMO radar system.

Our paper provides two main contributions: (i) We derive the first rigorous mathematical theory for the detection of moving targets in the azimuth-range-Doppler domain for random sensor arrays. (ii) The transmitted waveforms satisfy a variety of properties that are very desirable and important from a practical viewpoint. In particular, we show that Kerdock sequences, which would perform very poorly in single-antenna radar, are nearly ideally suited for MIMO radar with randomly spaced antennas. Since Kerdock codes are polyphase sequences, they have excellent PAPR and they are easy to implement in hardware via a simple look-up table. Thus, our framework does not just lead to useful theoretical insights, but also has a very strong practical appeal.

1.1. Connections with prior work and innovations. Random sensor arrays have been around for half a century. The pioneering work 27, 28, by Lo contains a mathematical analysis of important specific characteristics of random arrays, such as sidelope behavior and antenna gain. There is extensive engineering literature that deals with random arrays in connection with phased array radar technology, e.g. see [11. Recently, Carin made an explicit connection between the areas of random sensor arrays and compressive sensing [6]. He has shown that algorithms developed in these two seemingly different areas are in fact highly inter-related. The setup in [6] is quite different from ours, since the paper is only concerned with angular resolution (thus transmission waveforms do not even explicitly enter into the model), while it is often crucial in practice to be able to estimate range and Doppler as well. Moreover, the theoretical analysis in [6] follows more an engineering style and places less emphasis on mathematical rigor. The paper [7] provides interesting results for the angular estimation of stationary targets. Its setup is similar to that in [6, and quite different from ours, as it does not deal with waveform design nor with moving targets.

Kerdock codes have been proposed for radar in 20. However in the setting of a single transmit antenna. Kerdock codes are known to perform rather poorly

\footnotetext{
${ }^{2} \mathrm{~A}$ polyphase sequence is a sequence whose coefficients are of the form $e^{2 \pi i t_{k} / p}$ for some $t_{k} \in\{0, \ldots, p-1\}$, see e.g. 13 .

${ }^{3}$ The peak-to-aver power ratio of a signal $\mathbf{s}$ is defined, up to different normalizations, as $\frac{\|\mathbf{s}\|_{\infty}}{\|\mathbf{s}\|_{2}}$.

${ }^{4}$ This poor performance is caused by Property (ii) in Theorem 3.1
} 
even in the case of single targets as considered in [20. Only in the setting of mulitple transmit antennas can Kerdock codes exhibit their enormous potential. Our paper utilizes some properties of Kerdock codes proved in [20, but otherwise there is no overlap. In our paper we also present an extension of the main result, that allows for instance the use of the so-called finite harmonic oscillator system as transmission waveforms. These sequences have been derived in [14, where the authors also briefly sketch their use in a single-antenna radar system for the simple case of a single target. Thus, while our framework allows one to employ the finite harmonic oscillator system, there is essentially no overlap of our results with those in [14.

The paper 33 (coauthored by one of the authors) is closest to this paper, but the setting is in a sense complementary. 33] considers a MIMO radar setting with a very specific (non-random) choice for the antenna locations, but random waveforms, while the current paper deals with randomly spaced antennas, but very specific, deterministic waveforms. At first glance, the difference may appear to be mainly semantic. But in practice, the second setting has many advantages. From an engineer's viewpoint random waveforms have several drawback over properly designed deterministic waveforms: they are much harder to implement on a digital device (requiring more complicated hardware, more memory, ...); and they exhibit a larger peak-to-average-power ratio. On the other hand it makes no difference from the viewpoint of physics or hardware, if we place the antennas at random or at deterministic locations. In particular, the current paper yields some important insights, which cannot be inferred from [33]: We obtain a theoretical framework for radar operating with random antenna arrays, a technique which have been around for half a century; we show that Kerdock sequences, which are not useful for SISO or SIMO rada15, are excellent for MIMO radar; our approach allows for waveforms that satisfy a number of properties which are very desirable in practice, and are not satisfied by random waveforms. Indeed, as mentioned above, we also show that the finite harmonic oscillator system "plays well" with random antenna arrays.

Our paper is organized as follows. Section 2 describes the problem setup and the radar model. We review key properties of Kerdock codes in Section 3 Our main theorem is presented in Section 4 and Section 5 is devoted to the proof of the main theorem. In Section [ 6 we extend our framework to other deterministic waveforms, such as the finite harmonic oscillator system. Numerical simulations are presented in Section 7

1.2. Notation. For a matrix $\mathbf{A}$, we use $\mathbf{A}^{*}$ to denote its adjoint matrix, which is its conjugate transpose. The operator norm of $\mathbf{A}$ is the largest singular value of $\mathbf{A}$ and is denoted by $\|\mathbf{A}\|_{\text {op }}$. We denote the $k$-th column of $\mathbf{A}$ by $\mathbf{A}_{k}$ and the element in the $i$-th row and $k$-th column by $\mathbf{A}_{[i, k]}$. The coherence of $\mathbf{A}$ is defined as

$$
\mu(\mathbf{A}):=\max _{k \neq l} \frac{\left|\left\langle\mathbf{A}_{k}, \mathbf{A}_{l}\right\rangle\right|}{\left\|\mathbf{A}_{k}\right\|_{2}\left\|\mathbf{A}_{l}\right\|_{2}} .
$$

The $n \times n$ Discrete Fourier Transform (DFT) matrix is written as $\mathbf{F}_{n}$ and the $n \times n$ identity matrix as $\mathbf{I}_{n}$. For $\mathbf{x} \in \mathbb{C}^{n}$, let $\mathbf{T}_{\tau}$ denote the circulant translation

\footnotetext{
${ }^{5}$ SISO stands for single-input-single-output radar, and SIMO for single-input-multiple-output radar (i.e., a radar with one transmit and multiple receive antennas).
} 
operator, defined by

$$
\mathbf{T}_{\tau} \mathbf{x}(l)=\mathbf{x}(l-\tau), \quad \tau \in \mathbb{C}^{n},
$$

where $l-\tau$ is understood modulo $n$, and let $\mathbf{M}_{f}$ be the modulation operator defined by

$$
\mathbf{M}_{f} \mathbf{x}(l)=\mathbf{x}(l) e^{2 \pi i f l / n} .
$$

Acknowledgements. The authors acknowledge generous support by the National Science Foundation under grant dtra-dms 1042939 and by DARPA under grant N66001-11-1-4090.

\section{Problem Setup}

We consider a MIMO radar employing $N_{T}$ antennas at the transmitter and $N_{R}$ antennas at the receiver. We assume for convenience that transmitters and receivers are co-located, cf. Figure 1. Furthermore, we assume a coherent propagation scenario, i.e., the element spacing is sufficiently small so that the radar return from a given scatterer is fully correlated across the array. The arrays and all the scatterers are assumed to be in the same 2-D plane. The extension to the 3 - $\mathrm{D}$ case is straightforward.

The array manifolds $\mathbf{a}_{T}(\beta), \mathbf{a}_{R}(\beta)$ with randomly spaced antennas are given by

$$
\mathbf{a}_{T}(\beta)=\left[e^{2 \pi i p_{1} \beta}, e^{2 \pi i p_{2} \beta}, \ldots, e^{2 \pi i p_{N_{T}} \beta}\right]^{T},
$$

and

$$
\mathbf{a}_{R}(\beta)=\left[e^{2 \pi i q_{1} \beta}, e^{2 \pi i q_{2} \beta}, \ldots, e^{2 \pi i q_{N_{R}} \beta}\right]^{T},
$$

where we assume that the relative antenna spacings $p_{j}$ 's and $q_{j}$ 's are i.i.d. uniformly on $\left[0, \frac{N_{R} N_{T}}{2}\right]$. The $j$-th transmit antenna repeatedly transmits the signal $s_{j}(t)$, which is assumed to be a periodic, continuous-time signal of period-duration $T$ seconds and bandwidth $B$. We observe the back-scattered signal over a duration $T$, and since its bandwidth is $B$, it suffices that each receive antennas takes $N_{s}$ samples, where $N_{s}=T / \Delta_{s}$ and $\Delta_{s}=\frac{1}{2 B}$. It is convenient to introduce the finite-length vector $\mathbf{s}_{j}$ associated with $s_{j}$, via $\mathbf{s}_{j}(l):=s_{j}\left(l \Delta_{s}\right), l=1, \ldots, N_{s}$.

Let $\mathbf{Z}(t ; \beta, \tau, f)$ be the $N_{R} \times N_{s}$ noise-free received signal matrix from a unit strength target at direction $\beta$, delay $\tau$, and Doppler $f$ (corresponding to its radial velocity with respect to the radar). Then

$$
\mathbf{Z}(t ; \beta, \tau, f)=\mathbf{a}_{R}(\beta) \mathbf{a}_{T}^{T}(\beta) \mathbf{S}_{\tau, f}^{T},
$$

where $\mathbf{S}_{\tau, f}$ is a $N_{s} \times N_{T}$ matrix whose columns are the circularly delayed and Doppler shifted signals $s_{j}(t-\tau) e^{2 \pi i f t}$.

We let $\mathbf{z}(t ; \beta, \tau, f)=\operatorname{vec}\{\mathbf{Z}\}(t ; \beta, \tau, f)$ be the noise-free vectorized received signal. We set up a discrete azimuth-range-Doppler grid $\left\{\beta_{l}, \tau_{j}, f_{k}\right\}$ for $1 \leq l \leq N_{\beta}, 1 \leq j \leq$ $N_{\tau}$ and $1 \leq k \leq N_{f}$, where $\Delta_{\beta}, \Delta_{\tau}$ and $\Delta_{f}$ denote the corresponding discretization stepsizes. Using vectors $\mathbf{z}\left(t ; \beta_{l}, \tau_{j}, f_{k}\right)$ for all grid points $\left(\beta_{l}, \tau_{j}, f_{k}\right)$ we construct a complete response matrix $\mathbf{A}$ whose columns are $\mathbf{z}\left(t ; \beta_{l}, \tau_{j}, f_{k}\right)$ for $1 \leq l \leq N_{\beta}$ and $1 \leq j \leq N_{\tau}, 1 \leq k \leq N_{f}$. In other words, $\mathbf{A}$ is a $N_{R} N_{s} \times N_{\tau} N_{\beta} N_{f}$ matrix with columns

$$
\mathbf{A}_{\beta, \tau, f}=\mathbf{a}_{R}(\beta) \otimes \mathbf{S}_{\tau, f} \mathbf{a}_{T}(\beta) .
$$

\footnotetext{
${ }^{6}$ Actually the received signal will have a somwhat larger bandwidth $B_{1}>B$ due to the Doppler effect. However, in practice this increase in bandwidth is small, so we can assume $B \approx B_{1}$.
} 
Assume that the radar illuminates a scene consisting of $S$ scatterers located on $S$ points of the $\left(\beta_{l}, \tau_{j}, f_{k}\right)$-grid. Let $\mathbf{x}$ be a sparse vector whose non-zero elements are the complex amplitudes of the scatterers in the scene. The zero elements correspond to grid points which are not occupied by scatterers. We can then define the radar signal $\mathbf{y}$ received from this scene by

$$
\mathbf{y}=\mathbf{A x}+\mathbf{w}
$$

where $\mathbf{y}$ is an $N_{R} N_{s} \times 1$ vector, $\mathbf{x}$ is an $N_{\tau} N_{\beta} N_{f} \times 1$ sparse vector and $\mathbf{w}$ is an $N_{R} N_{s} \times 1$ complex Gaussian noise vector. Our goal is to solve for $\mathbf{x}$, i.e., to locate the scatterers (and their reflection coefficients) in the azimuth-delay-Doppler domain.

Remark: The assumption that the targets lie on the grid points, while common in compressive sensing, is certainly restrictive. A violation of this assumption will result in a model mismatch, sometimes dubbed gridding error, which can potentially be quite severe [18, 8]. Recently some interesting strategies have been proposed to overcome this gridding error [10, 35. But these methods - at least in their current form - are not directly applicable to our setting. This model mismatch issue is beyond the scope of this paper and will be addressed in our future research.

\section{KERDOCK CODES}

In this section we introduce one particularly useful set of transmission waveforms. Due to the setup in Section 2 it suffices that we deal with discrete, finite-length signals as transmission waveforms. We briefly review the construction of Kerdock codes and some of their fundamental properties. There is a long list of properties that radar waveforms should satisfy. As we will see in this paper, Kerdock codes fulfill many of them. Kerdock codes over $\mathbb{Z}_{2}$ (i.e., binary Kerdock codes) were originally introduced in $[23$. In the seminal paper [4 the authors extend Kerdock codes from $\mathbb{Z}_{2}$ to $\mathbb{Z}_{4}$. By doing so, they uncover many fascinating properties of Kerdock codes and reveal numerous deep connections between coding theory, discrete geometry and group theory. In the same paper, the authors also extend Kerdock codes to the setting of $\mathbb{Z}_{p}$, where $p$ is an odd prime.

Kerdock codes are an example of so-called mutually unbiased bases [39, 34. Kerdock codes have also been proposed for use in communications engineering [16, 22. In 20] the authors suggest the use of Kerdock codes for radar, based on the peculiar properties of the discrete ambiguity function associated with Kerdock codes. We emphasize however that for the single transmit antenna radar scenario Kerdock codes would actually perform rather badly, as discussed after Theorem 3.1 and shown by Figure 5 in Section 7 It is only in the setting of multiple transmit antennas that Kerdock codes become useful for radar.

For the remainder of this paper we will only be concerned with Kerdock codes over $\mathbb{Z}_{p}$. Some of the Kerdock codes over $\mathbb{Z}_{p}$, namely those corresponding to desarguesian planes in the language of [4, have also been derived earlier in [25] and 24]. A simple way to construct these Kerdock codes is the following, in which they arise as eigenvectors of time-frequency shift operators. Let $p$ be an odd prime number. For each $k=0, \ldots, p-1$ we compute the eigenvector decomposition of $\mathbf{T}_{0} \mathbf{M}_{k}$ (which always exists, since $\mathbf{T}_{0} \mathbf{M}_{k}$ is a unitary matrix)

$$
\mathbf{U}_{(k)} \boldsymbol{\Sigma}_{(k)} \mathbf{U}_{(k)}^{*}=\mathbf{T}_{0} \mathbf{M}_{k},
$$


where the unitary matrix $\mathbf{U}_{(k)}$ contains the eigenvectors of $\mathbf{T}_{0} \mathbf{M}_{k}$ and the diagonal matrix $\boldsymbol{\Sigma}_{(k)}$ the associated eigenvalue: 7 . Furthermore, we define $\mathbf{U}_{(p)}:=I_{p}$. Now, let $\mathbf{u}_{k, j}$ be the $j$-th column of $\mathbf{U}_{(k)}$. The set consisting of the $p^{2}+p$ vectors $\left\{\mathbf{u}_{k, j}, k=0, \ldots, p ; j=0, \ldots, p-1\right\}$ forms a $\mathbb{Z}_{p}$-Kerdock code. There are numerous equivalent ways to derive this Kerdock code, but, as pointed out earlier, not all Kerdock codes over $\mathbb{Z}_{p}$ are equivalent (see also the comment following Corollary 11.6 in [4). But we will be a bit sloppy, and simply refer to the Kerdock code constructed above as the Kerdock code.

In the following theorem we collect those key properties of Kerdock codes that are most relevant for radar. These properties are either explicitly proved in [4, 20] or can be derived easily from properties stated in those papers.

Theorem 3.1. Kerdock codes over $\mathbb{Z}_{p}$, where $p$ is an odd prime, satisfy the following properties:

(i) Mutually unbiased bases: For all $k=0, \ldots, p$ and all $j=0, \ldots, p-1$, there holds:

$$
\left|\left\langle\mathbf{u}_{k, j}, \mathbf{u}_{k^{\prime}, j^{\prime}}\right\rangle\right|= \begin{cases}1 & \text { if } k=k^{\prime}, j=j^{\prime}, \\ 0 & \text { if } k=k^{\prime}, j \neq j^{\prime}, \\ \frac{1}{\sqrt{p}} & \text { if } k \neq k^{\prime} .\end{cases}
$$

(ii) Time-frequency "autocorrelation":

(a) For any fixed $(f, l) \neq(0,0)$ there exists a unique $k_{0}$ such that

$$
\begin{aligned}
\left|\left\langle\mathbf{M}_{f} \mathbf{T}_{l} \mathbf{u}_{k_{0}, j}, \mathbf{u}_{k_{0}, j}\right\rangle\right| & =1 \quad \text { for } j=0, \ldots, p-1, \\
\left|\left\langle\mathbf{M}_{f} \mathbf{T}_{l} \mathbf{u}_{k, j}, \mathbf{u}_{k, j}\right\rangle\right| & =0 \quad \text { for } k \neq k_{0} .
\end{aligned}
$$

(b) For any fixed $0 \leq k \leq p-1$, there exist $\left(f_{r}, l_{r}\right), r=1, \ldots, p$ such that

$$
\left|\left\langle\mathbf{M}_{f_{r}} \mathbf{T}_{l_{r}} \mathbf{u}_{k, j}, \mathbf{u}_{k, j}\right\rangle\right|=1 \quad \text { for } j=0, \ldots, p-1,
$$

(iii) Time-frequency crosscorrelation: For all $k \neq k^{\prime}$ and all $f$ and $l$ there holds:

$$
\left|\left\langle\mathbf{M}_{f} \mathbf{T}_{l} \mathbf{u}_{k, j}, \mathbf{u}_{k^{\prime}, j}\right\rangle\right| \leq \frac{1}{\sqrt{p}} \quad \text { for } j=0, \ldots, p-1 .
$$

(iv) Polyphase property (Roots of unity property) in time and in frequency: For any $k=0, \ldots, p-1 ; j=0, \ldots, p-1$, there holds:

$$
\mathbf{u}_{k, j}(l)=e^{2 \pi i r / p} \quad \text { for some } r \in\{0, \ldots, p-1\} .
$$

For any $k=1, \ldots, p ; j=0, \ldots, p-1$, there holds:

$$
\hat{\mathbf{u}}_{k, j}(l)=e^{2 \pi i r / p} \quad \text { for some } r \in\{0, \ldots, p-1\} .
$$

Proof. Property (i) is proved for instance in Lemma 11.3 in [4. Properties (ii) and (iii) appear in Theorem 3 of [20. Statement (17) of property (iv) follows from the comment right after Corollary 11.6 in 4. Finally, statement (18) of property (iv) follows from (12) together with property (3) and the well-known fundamental relationships

$$
\mathbf{F}_{p} \mathbf{T}_{x} \mathbf{F}_{p}^{*}=\mathbf{M}_{-x}, \quad \mathbf{F}_{p} \mathbf{M}_{x} \mathbf{F}_{p}^{*}=\mathbf{T}_{x} .
$$

\footnotetext{
${ }^{7}$ The attentive reader will have noticed that $\mathbf{U}_{(0)}$ is just the $p \times p$ DFT matrix $\mathbf{F}_{p}$.
} 
Kerdock codes have been proposed for adaptive radar in [20. We emphasize again though that Kerdock codes would not be very effective for a radar system with a single transmit antenna (SISO or SIMO radar). This can be easily seen as follows: Assume we only have one antenna that transmits one waveform s. Because of (15), $\mathbf{s}$ is (up to a constant phase factor) equal to $\mathbf{M}_{f} \mathbf{T}_{l} \mathbf{s}$ for some $(f, l)$. In practice this ambiguity prevents us from determining the distance and the velocity of the object, when using Kerdock codes for SISO or SIMO.

As a consequence of the aforementioned ambiguity we will not use all of the Kerdock codes as transmission signals for our MIMO radar, instead we will choose one code for each index $k$. The reason is that we need the waveforms to have low time-frequency crosscorrelation, while (16) only holds when $k$ and $k^{\prime}$ are different.

Definition 3.2 (Kerdock waveforms). Let $\left\{\mathbf{u}_{k, j}, k=0, \ldots, p, j=0, \ldots, p-1\right\}$ be a Kerdock code over $\mathbb{Z}_{p}$. The Kerdock waveforms $\mathbf{k}_{0}, \ldots, \mathbf{k}_{r}$, where $r<p$, are given by $\mathbf{k}_{k}=\mathbf{u}_{k, j}$ for some arbitrary $j$. In other words, for each $k=0, \ldots, r-1$ we pick an arbitrary vector from the orthonormal basis $\left\{\mathbf{u}_{k, j}\right\}_{j=0}^{p-1}$.

Note that Kerdock waveforms do not include any unit vectors, since only the first $r$ unitary matrices $\mathbf{U}_{(0)}, \ldots, \mathbf{U}_{(r-1)}$ are considered and $r$ is strictly less than $p$ (recall that $\mathbf{U}_{(p)}=\mathbf{I}_{p}$ ).

\section{THE MAIN RESULT}

As mentioned in the introduction, a standard approach to solve (11) when $x$ is sparse, is

$$
\min _{\mathbf{x}} \frac{1}{2}\|\mathbf{A x}-\mathbf{y}\|_{2}^{2}+\lambda\|\mathbf{x}\|_{1},
$$

which is also known as lasso 36. But instead of (19), we will use the debiased lasso. That means first we compute an approximation $\tilde{I}$ for the support of $\mathbf{x}$ by solving (19). This is the detection step. Then, in the estimation step, we "debias" the solution by computing the amplitudes of $\mathbf{x}$ via solving the reduced-size least squares problem min $\left\|\mathbf{A}_{\tilde{I}} \mathbf{x}_{\tilde{I}}-\mathbf{y}\right\|_{2}$, where $\mathbf{A}_{\tilde{I}}$ is the submatrix of $\mathbf{A}$ consisting of the columns corresponding to the index set $\tilde{I}$, and similarly for $\mathbf{x}_{\tilde{I}}$.

We assume that the locations of the targets are random. To be precise, we assume that the $S$ nonzero coefficients of $x$ are selected uniformly at random and the phases of the non-zero entries of $x$ are random and uniformly distributed in $[0,2 \pi)$. We will refer to this model as the generic $S$-sparse model.

We are now ready to state our main result.

Theorem 4.1. Consider $\mathbf{y}=\mathbf{A} \mathbf{x}+\mathbf{w}$, where $\mathbf{A}$ is defined as in (10) and $\mathbf{w}_{j} \in$ $\mathcal{C} N\left(0, \sigma^{2}\right)$. Assume that the positions of the transmit and receive antennas $p_{j}$ 's and $q_{j}$ 's are chosen i.i.d. uniformly on $\left[0, \frac{N_{R} N_{T}}{2}\right]$ at random. Suppose further that each transmit antenna sends a different Kerdock waveform, i.e. the columns of the signal matrix $\mathbf{S}$ are different Kerdock waveforms. Choose the discretization stepsizes to be $\Delta_{\beta}=\frac{2}{N_{R} N_{T}}, \Delta_{\tau}=\frac{1}{2 B}, \Delta_{f}=\frac{1}{T}$ and suppose that

$$
\max \left(N_{R} N_{T}, 32 N_{T}^{3} \log N_{\tau} N_{f} N_{\beta}\right) \leq N_{s}=N_{\tau},
$$

and also

$$
\log ^{2} N_{\tau} N_{f} N_{\beta} \leq N_{T} \leq N_{R}
$$


If $\mathbf{x}$ is drawn from the generic $S$-sparse scatterer model with

$$
S \leq \frac{c_{0} N_{\tau}}{\log N_{\tau} N_{f} N_{\beta}}
$$

for some constant $c_{0}>0$, and if

$$
\min _{k \in I}\left|\mathbf{x}_{k}\right|>\frac{8 \sqrt{3} \sigma}{\sqrt{N_{R} N_{T}}} \sqrt{2 \log N_{\tau} N_{f} N_{\beta}},
$$

then the solution $\tilde{\mathbf{x}}$ of the debiased lasso computed with $\lambda=2 \sigma \sqrt{2 \log N_{\tau} N_{f} N_{\beta}}$ satisfies

$$
\operatorname{supp}(\tilde{\mathbf{x}})=\operatorname{supp}(\mathbf{x})
$$

with probability at least

$$
1-p_{1}
$$

and

$$
\frac{\|\tilde{\mathbf{x}}-\mathbf{x}\|_{2}}{\|\mathbf{x}\|_{2}} \leq \frac{5 \sigma \sqrt{3 N_{R} N_{s}}}{\|\mathbf{y}\|_{2}}
$$

with probability at least

$$
\left(1-p_{1}\right)\left(1-p_{2}\right)
$$

where

$$
\begin{aligned}
p_{1}=16 N_{\tau}^{-2} N_{R}^{-1} & +8 N_{\tau}^{-2} N_{f}^{-2}+4 N_{T} N_{\tau}^{-2} N_{f}^{-2}+4\left(N_{\tau} N_{f}\right)^{-1} \\
+ & 4 N_{\tau}^{-3} N_{f}^{-3} N_{R}^{-2} N_{T}^{-1}+8 N_{T}^{-2}\left(N_{\tau} N_{f} N_{R}\right)^{-3},
\end{aligned}
$$

and

$p_{2}=2\left(N_{\tau} N_{f} N_{\beta}\right)^{-1}\left(2 \pi \log \left(N_{\tau} N_{f} N_{\beta}\right)+K\left(N_{\tau} N_{f} N_{\beta}\right)^{-1}\right)+\mathcal{O}\left(\left(N_{\tau} N_{f} N_{\beta}\right)^{-2 \log 2}\right)$.

\section{Remarks:}

(1) The condition $N_{T} \leq N_{R}$ in (21) is by no means necessary, but rather to make our computation a little cleaner. We could change it into $N_{T} \leq 2 N_{R}$, then the theorem would remain true with a slightly different probability of success.

(2) It may seem that the conditions in (20) and (21) are a bit restrictive. But, in practice, our method works with a broad range of parameters as the simulations show in Section 7

\section{Proof of the Result}

To prove Theorem 4.1, we use a theorem by Candès and Plan (Theorem 1.3 in [5]) which requires to estimate the operator norm of $\mathbf{A}$ and the coherence of A. The original theorem only treats the real-valued case, it can be extended to complex-values case after some straightforward modifications (see Appendix B in [33). 
5.1. Auxiliary results. We first need the following Bernstein type lemma.

Lemma 5.1. Suppose $M$ is an $m \times m$ matrix, $\alpha$ and $\beta$ are two joint independent random vectors in $\mathbb{C}^{m}$ with zero means and $\left|\alpha_{k}\right|=\left|\beta_{k}\right|=1$ for $k=1, \ldots, m$. If $n$ is a positive constant, then for any $t>0$ and $s>0$,

(1) if $\left|m_{k j}\right| \leq \frac{1}{\sqrt{n}}$ for all $k, j$, then

$$
\mathbb{P}(|\langle M \alpha, \beta\rangle| \leq m t) \geq 1-4 m \exp \left(-\frac{t^{2}}{4 \frac{m}{n}}\right) .
$$

and

$$
\mathbb{P}(|\langle M \alpha, \alpha\rangle| \leq 2 m t) \geq 1-8 m \exp \left(-\frac{t^{2}}{2 \frac{m}{n}}\right),
$$

(2) if $\left|m_{k j}\right| \leq \frac{1}{\sqrt{n}}$ for $k \neq j$ and $m_{j j}=1$, then

$$
\begin{aligned}
& \mathbb{P}(|\langle M \alpha, \beta\rangle| \leq s+m t) \geq 1-4 \exp \left(-\frac{s^{2}}{4 m}\right)-4 m \exp \left(-\frac{t^{2}}{4 \frac{m}{n}}\right), \\
& \text { and } \\
& \qquad \mathbb{P}(m(1-2 t) \leq|\langle M \alpha, \alpha\rangle| \leq m(1+2 t)) \geq 1-8 m \exp \left(-\frac{t^{2}}{2 \frac{m}{n}}\right) .
\end{aligned}
$$

Proof.

$$
\begin{aligned}
\langle M \alpha, \beta\rangle & =\sum_{k, j=1}^{m} m_{k j} \alpha_{j} \bar{\beta}_{k} \\
& =\sum_{l=1}^{m} \sum_{j=1}^{m} m_{j \oplus l, j} \alpha_{j} \bar{\beta}_{j \oplus l},
\end{aligned}
$$

where $\oplus$ denotes addition modulo $m$.

Let us first assume that $\left|m_{k j}\right| \leq \frac{1}{\sqrt{n}}$.

Since $\alpha$ and $\beta$ are joint independent, then for any $l$, the entries in $\sum_{j=1}^{m} m_{j \oplus l, j} \alpha_{j} \bar{\beta}_{j \oplus l}$ are all joint independent and it is easy to check that $\mathbb{E}\left(m_{j \oplus l, j} \alpha_{j} \bar{\beta}_{j \oplus l}\right)=0$ and $\left|m_{j \oplus l, j} \alpha_{j} \bar{\beta}_{j \oplus l}\right|=\left|m_{j \oplus l, j}\right|$, then Theorem 4.5 in 21] will give,

$$
\begin{aligned}
\mathbb{P}\left(\left|\sum_{j=1}^{m} m_{j \oplus l, j} \alpha_{j} \bar{\beta}_{j \oplus l}\right| \leq t\right) & \geq 1-4 \exp \left(-\frac{t^{2}}{4 \sum_{j}\left|m_{j \oplus l, j}\right|^{2}}\right) \\
& \geq 1-4 \exp \left(-\frac{t^{2}}{4 \frac{m}{n}}\right) .
\end{aligned}
$$

We take all $m$ different choices of $l$, then

$$
\mathbb{P}\left(\left|\sum_{l=1}^{m} \sum_{j=1}^{m} m_{i \oplus l, j} \alpha_{j} \bar{\beta}_{j \oplus l}\right| \leq m t\right) \geq 1-4 m \exp \left(-\frac{t^{2}}{4 \frac{m}{n}}\right),
$$

which proves (28).

$$
\langle M \alpha, \alpha\rangle=\sum_{l=1}^{m} \sum_{j=1}^{m} m_{j \oplus l, j} \alpha_{j} \bar{\alpha}_{j \oplus l}
$$


THOMAS STROHMER AND HAICHAO WANG

different from above, the entries in $\sum_{j=1}^{m} m_{j \oplus l, j} \alpha_{j} \bar{\alpha}_{j \oplus l}$ are no longer all jointly independent. But similar to the proof of Theorem 5.1 in 30] and Lemma 3 in [33], we observe that for any $l$ we can split the index set $1, \ldots, m$ into two subsets $T_{l}^{1}, T_{l}^{2} \subset\{1, \ldots, m\}$, each of size $m / 2$, such that the $m / 2$ variables $\alpha_{j} \bar{\alpha}_{j \oplus l}$ are jointly independent for $j \in T_{l}^{1}$, and analogous for $T_{l}^{2}$. (For convenience we assume here that $m$ is even, but with a negligible modification the argument also applies for odd $m$.) In other words, each of the sums $\sum_{j \in T_{l}^{r}} m_{j \oplus l, j} \alpha_{j} \bar{\alpha}_{j \oplus l}, r=1,2$, contains only jointly independent terms.

So for each $l$,

$$
\mathbb{P}\left(\left|\sum_{j \in T_{l}^{r}} m_{j \oplus l, j} \alpha_{j} \bar{\alpha}_{j \oplus l}\right| \leq t\right) \geq 1-4 \exp \left(-\frac{t^{2}}{2 \frac{m}{n}}\right),
$$

which implies that

$$
\mathbb{P}\left(\left|\sum_{j} m_{j \oplus l, j} \alpha_{j} \bar{\alpha}_{j \oplus l}\right| \leq 2 t\right) \geq 1-8 \exp \left(-\frac{t^{2}}{2 \frac{m}{n}}\right),
$$

Again, we take all $m$ different choices of $l$, then

$$
\mathbb{P}\left(\left|\sum_{l=1}^{m} \sum_{j=1}^{m} m_{j \oplus l, j} \alpha_{j} \bar{\alpha}_{j \oplus l}\right| \leq 2 m t\right) \geq 1-8 m \exp \left(-\frac{t^{2}}{2 \frac{m}{n}}\right),
$$

which proves (29).

Now let us assume that $\left|m_{k j}\right| \leq \frac{1}{\sqrt{n}}$ for $k \neq j$ and $m_{j j}=1$.

$$
\begin{aligned}
\langle M \alpha, \beta\rangle & =\sum_{j=1}^{m} m_{j j} \alpha_{j} \bar{\beta}_{j}+\sum_{l=1}^{m-1} \sum_{j=1}^{m} m_{j \oplus l, j} \alpha_{j} \bar{\beta}_{j \oplus l} \\
& =\sum_{j=1}^{m} \alpha_{j} \bar{\beta}_{j}+\sum_{l=1}^{m-1} \sum_{j=1}^{m} m_{j \oplus l, j} \alpha_{j} \bar{\beta}_{j \oplus l} .
\end{aligned}
$$

Since $\alpha$ and $\beta$ are joint independent and $\left|\alpha_{j} \bar{\beta}_{j}\right|=1$,

$$
\mathbb{P}\left(\left|\sum_{j=1}^{m} \alpha_{j} \bar{\beta}_{j}\right| \leq s\right) \geq 1-4 \exp \left(-\frac{s^{2}}{4 m}\right) .
$$

Similar to the proof of (33) above, we have that

$$
\mathbb{P}\left(\left|\sum_{l=1}^{m-1} \sum_{j=1}^{m} m_{j \oplus l, j} \alpha_{j} \bar{\beta}_{j \oplus l}\right| \leq(m-1) t\right) \geq 1-4(m-1) \exp \left(-\frac{t^{2}}{4 \frac{m}{n}}\right),
$$

together with (37), it follows

$$
\mathbb{P}(|\langle M \alpha, \beta\rangle| \leq s+(m-1) t) \geq 1-4 \exp \left(-\frac{s^{2}}{4 m}\right)-4(m-1) \exp \left(-\frac{t^{2}}{4 \frac{m}{n}}\right),
$$

which proves (30).

$$
\langle M \alpha, \alpha\rangle=\sum_{j=1}^{m} m_{j j}+\sum_{l=1}^{m-1} \sum_{j=1}^{m} m_{j \oplus l, j} \alpha_{j} \bar{\alpha}_{j \oplus l}=m+\sum_{l=1}^{m-1} \sum_{j=1}^{m} m_{j \oplus l, j} \alpha_{j} \bar{\alpha}_{j \oplus l},
$$


then (31) results from similar proof as for (29) and the triangle inequality.

\subsection{Estimation of the Operator Norm.}

Lemma 5.2. Let $\mathbf{A}$ be the matrix in Theorem 4.1 satisfying (20). Then

$$
\mathbb{P}\left(\|\mathbf{A}\|_{o p}^{2} \leq 2 N_{f} N_{R}^{2} N_{T}^{2}\right) \geq 1-8 N_{\tau}^{-2} N_{R}^{-1} .
$$

Proof. Since $\|\mathbf{A}\|_{\text {op }}^{2}=\left\|\mathbf{A} \mathbf{A}^{*}\right\|_{\text {op }}$, we consider matrix $\mathbf{B}=\mathbf{A} \mathbf{A}^{*}$ as block matrix

$$
\left[\begin{array}{cccc}
\mathbf{B}_{1,1} & \mathbf{B}_{1,2} & \ldots & \mathbf{B}_{1, N_{R}} \\
\vdots & \ddots & & \vdots \\
\mathbf{B}_{N_{R}, 1} & \cdots & & \mathbf{B}_{N_{R}, N_{R}}
\end{array}\right],
$$

where the blocks $\left\{\mathbf{B}_{j, j^{\prime}}\right\}_{j, j^{\prime}=1}^{N_{R}}$ are matrices of size $N_{t} \times N_{t}$.

Via a simple permutation, we can turn $\mathbf{B}$ into a matrix $\mathbf{C}$ with blocks $\left\{\mathbf{C}_{l, l^{\prime}}\right\}_{l, l^{\prime}=1}^{N_{s}}$ of size $N_{R} \times N_{R}$, where the $\left(j, j^{\prime}\right)$-th entry of the block $\mathbf{C}_{l, l^{\prime}}$ is defined as

$$
\begin{aligned}
& \mathbf{C}_{\left[l, j ; l^{\prime} j^{\prime}\right]}=\mathbf{B}_{\left[j, l ; j^{\prime}, l^{\prime}\right]}=\left(\mathbf{A} \mathbf{A}^{*}\right)_{\left[j, l ; j^{\prime}, l^{\prime}\right]}=\sum_{\beta} \sum_{\tau} \sum_{f} \mathbf{A}_{[j, l ; \tau, f, \beta]} \overline{\mathbf{A}_{\left[j^{\prime}, l^{\prime} ; \tau, f, \beta\right]}} \\
& =\sum_{\beta} e^{2 \pi i\left(q_{j}-q_{j^{\prime}}\right) \beta} \sum_{k=1}^{N_{T}} \sum_{k^{\prime}=1}^{N_{T}} e^{2 \pi i\left(p_{k}-p_{k^{\prime}}\right) \beta}\left\langle\mathbf{T}_{l} \mathbf{k}_{k}, \mathbf{T}_{l^{\prime}} \mathbf{k}_{k^{\prime}}\right\rangle \sum_{m=1}^{N_{f}} e^{2 \pi i\left(l-l^{\prime}\right) \Delta_{t} m \Delta_{f}} \\
& =N_{f} \delta_{l, l^{\prime}} \sum_{\beta} e^{2 \pi i\left(q_{j}-q_{j^{\prime}}\right) \beta} \sum_{k=1}^{N_{T}} \sum_{k^{\prime}=1}^{N_{T}} e^{2 \pi i\left(p_{k}-p_{k^{\prime}}\right) \beta}\left\langle\mathbf{T}_{l} \mathbf{k}_{k}, \mathbf{T}_{l^{\prime}} \mathbf{k}_{k^{\prime}}\right\rangle
\end{aligned}
$$

Then it is easy to see that $\mathbf{C}$ is block-diagonal, and all the diagonal-blocks are identical. So we only have to bound the first block $\mathbf{C}_{1,1}$.

$$
\begin{aligned}
& \mathbf{C}_{\left[1, j ; 1, j^{\prime}\right]}=N_{f} \sum_{\beta} e^{2 \pi i\left(q_{j}-q_{j^{\prime}}\right) \beta} \sum_{k=1}^{N_{T}} \sum_{k^{\prime}=1}^{N_{T}} e^{2 \pi i\left(p_{k}-p_{k^{\prime}}\right) \beta}\left\langle\mathbf{k}_{k}, \mathbf{k}_{k^{\prime}}\right\rangle \\
& =N_{f} \sum_{n=0}^{N_{R} N_{T}-1} e^{2 \pi i\left(q_{j}-q_{j^{\prime}}\right) \frac{n}{N_{R^{N} T}}} \sum_{k=1}^{N_{T}} \sum_{k^{\prime}=1}^{N_{T}} e^{2 \pi i\left(p_{k}-p_{k^{\prime}}\right) \frac{n}{N_{R} N_{T}}}\left\langle\mathbf{k}_{k}, \mathbf{k}_{k^{\prime}}\right\rangle .
\end{aligned}
$$

Define $c_{n}=\sum_{k=1}^{N_{T}} \sum_{k^{\prime}=1}^{N_{T}} e^{2 \pi i\left(p_{k}-p_{k}^{\prime}\right)} \frac{n}{N_{R^{N} T}}\left\langle\mathbf{k}_{k}, \mathbf{k}_{k^{\prime}}\right\rangle$, then

$$
\mathbf{C}_{1,1}=N_{f} \sum_{n=0}^{N_{R} N_{T}-1} c_{n} X_{n},
$$

where $X_{n}$ is the matrix-valued random variable given by $\left(X_{n}\right)_{j, j^{\prime}}=e^{2 \pi i\left(q_{j}-q_{j^{\prime}}\right) \frac{n}{N_{R^{N} T}}}$ and therefore $\left\|X_{n}\right\|_{\mathrm{op}}=N_{R}$.

Note that $\mathbb{E}\left(e^{2 \pi i\left(p_{k}-p_{k^{\prime}}\right) n}\right)=0$ and $\left|\left\langle\mathbf{k}_{k}, \mathbf{k}_{k^{\prime}}\right\rangle\right| \leq \frac{1}{\sqrt{N_{s}}}$ for $k \neq k^{\prime}$. Choosing $t=2 \sqrt{\frac{N_{T}}{N_{s}}} \sqrt{\log N_{\tau} N_{R} N_{T}}$ in (31) of Lemma 5.1, we arrive at

$$
\mathbb{P}\left(\left|c_{n}\right| \leq N_{T}\left(1+4 \sqrt{\frac{N_{T}}{N_{s}}} \sqrt{\log N_{\tau} N_{R} N_{T}}\right)\right) \geq 1-8 N_{T}\left(N_{\tau} N_{R} N_{T}\right)^{-2},
$$


then the assumption in (20) implies that $16 N_{T} \log N_{\tau} N_{R} N_{T} \leq N_{s}$, therefore

$$
\mathbb{P}\left(\left|c_{n}\right| \leq 2 N_{T}\right) \geq 1-8 N_{T}\left(N_{\tau} N_{R} N_{T}\right)^{-2} .
$$

We apply the union bound over the $N_{R} N_{T}$ possibilities associated with $n$ and get

$$
\mathbb{P}\left(\max \left|c_{n}\right| \leq 2 N_{T}\right) \geq 1-8 N_{\tau}^{-2} N_{R}^{-1}
$$

which implies that

$$
\mathbb{P}\left(\left\|\mathbf{C}_{1,1}\right\|_{\text {op }} \leq 2 N_{f} N_{R}^{2} N_{T}^{2}\right) \geq 1-8 N_{\tau}^{-2} N_{R}^{-1} .
$$

Then the fact that $\|\mathbf{B}\|_{\text {op }}=\|\mathbf{C}\|_{\text {op }}=\left\|\mathbf{C}_{1,1}\right\|_{\text {op }}$ will give us the conclusion.

\subsection{Estimation of the Coherence.}

Lemma 5.3. Let $\mathbf{A}$ be the matrix in Theorem 4.1 satisfying (20) and (21). Then

$$
\max _{(\tau, f, \beta) \neq\left(\tau^{\prime}, f^{\prime}, \beta^{\prime}\right)}\left|\left\langle\mathbf{A}_{\tau, f, \beta}, \mathbf{A}_{\tau^{\prime}, f^{\prime}, \beta^{\prime}}\right\rangle\right| \leq 16 N_{R} \log N_{\tau} N_{f} N_{R} N_{T}
$$

with probability at least

$1-8 N_{\tau}^{-2} N_{f}^{-2}-4 N_{T} N_{\tau}^{-2} N_{f}^{-2}-4\left(N_{\tau} N_{f}\right)^{-1}-4 N_{\tau}^{-3} N_{f}^{-3} N_{R}^{-2} N_{T}^{-1}-8 N_{T}^{-2}\left(N_{\tau} N_{f} N_{R}\right)^{-3}$.

Proof. We need to find an upper bound for

$$
\max \left|\left\langle\mathbf{A}_{\tau, f, \beta}, \mathbf{A}_{\tau^{\prime}, f^{\prime}, \beta^{\prime}}\right\rangle\right| \quad \text { for }(\tau, f, \beta) \neq\left(\tau^{\prime}, f^{\prime}, \beta^{\prime}\right) .
$$

Recall the $\mathbf{S}_{\tau, f}=\mathbf{M}_{f} \mathbf{T}_{\tau} \mathbf{S}$, it follows from the definition that

$$
\mathbf{A}_{\tau, f, \beta}=\mathbf{a}_{R}(\beta) \otimes\left(\mathbf{S}_{\tau, f} \mathbf{a}_{T}(\beta)\right),
$$

from which we readily compute

$$
\left|\left\langle\mathbf{A}_{\tau, f, \beta}, \mathbf{A}_{\tau^{\prime}, f^{\prime}, \beta^{\prime}}\right\rangle\right|=\left|\left\langle\mathbf{a}_{R}(\beta), \mathbf{a}_{R}\left(\beta^{\prime}\right)\right\rangle\right|\left|\left\langle\mathbf{S}_{\tau, f} \mathbf{a}_{T}(\beta), \mathbf{S}_{\tau^{\prime}, f^{\prime}} \mathbf{a}_{T}\left(\beta^{\prime}\right)\right\rangle\right| .
$$

We use the discretization $\beta=n \Delta_{\beta}, \beta^{\prime}=n^{\prime} \Delta_{\beta}$, where $\Delta_{\beta}=\frac{2}{N_{R} N_{T}}, n, n^{\prime}=$ $1, \ldots, N_{\beta}$, with $N_{\beta}=N_{R} N_{T}$.

Since

$$
\left|\left\langle\mathbf{S}_{\tau, f} \mathbf{a}_{T}(\beta), \mathbf{S}_{\tau^{\prime}, f^{\prime}} \mathbf{a}_{T}\left(\beta^{\prime}\right)\right\rangle\right|=\left|\left\langle\mathbf{S}_{\tau-\tau^{\prime}, f-f^{\prime}} \mathbf{a}_{T}(\beta), \mathbf{S a}_{T}\left(\beta^{\prime}\right)\right\rangle\right|
$$

for $\tau, \tau^{\prime}=0, \ldots, N_{\tau}-1, f, f^{\prime}=0, \ldots, N_{f}-1$. We can confine the range of values for $\tau, \tau^{\prime}$ to $\tau^{\prime}=0, \tau=0, \ldots, N_{\tau}-1$ and $f, f^{\prime}$ to $f^{\prime}=0, f=0, \ldots, N_{f}-1$, then we only need to estimate $\left|\left\langle\mathbf{S}_{\tau, f} \mathbf{a}_{T}(\beta), \mathbf{S a}_{T}\left(\beta^{\prime}\right)\right\rangle\right|$. We now consider three cases.

Case (i) $\beta \neq \beta^{\prime}, \tau=0, f=0$ :

By Theorem 4.5 in [21], for any $t_{1}>0$

$$
\mathbb{P}\left(\left|\left\langle\mathbf{a}_{R}(\beta), \mathbf{a}_{R}\left(\beta^{\prime}\right)\right\rangle\right| \geq t_{1}\right) \leq 4 \exp \left(-\frac{t_{1}^{2}}{4 N_{R}}\right),
$$

choosing $t_{1}=2 \sqrt{2} \sqrt{N_{R}} \sqrt{\log N_{\tau} N_{f} N_{R} N_{T}}$ will give us that

$$
\mathbb{P}\left(\left|\left\langle\mathbf{a}_{R}(\beta), \mathbf{a}_{R}\left(\beta^{\prime}\right)\right\rangle\right| \leq 2 \sqrt{2} \sqrt{N_{R}} \sqrt{\log N_{\tau} N_{f} N_{R} N_{T}}\right) \geq 1-4\left(N_{\tau} N_{f} N_{R} N_{T}\right)^{-2} .
$$


Define $M=\mathbf{S}^{*} \mathbf{S}$ then $\left|m_{k j}\right|=\left|\left\langle\mathbf{k}_{k}, \mathbf{k}_{j}\right\rangle\right| \leq \frac{1}{\sqrt{N_{s}}}$ for $k \neq j$ and $m_{j j}=1$. We choose $s=2 \sqrt{2} \sqrt{N_{T}} \sqrt{\log N_{\tau} N_{f} N_{R} N_{T}}$ and $t=2 \sqrt{2} \sqrt{\frac{N_{T}}{N_{s}}} \sqrt{\log N_{\tau} N_{f} N_{R} N_{T}}$ in (30) of Lemma 5.1 and get

$$
\begin{aligned}
\mathbb{P}\left(\left|\left\langle\mathbf{S}^{*} \mathbf{S a}_{T}(\beta), \mathbf{a}_{T}\left(\beta^{\prime}\right)\right\rangle\right|\right. & \left.\leq\left(2 \sqrt{2} \sqrt{N_{T}}+2 \sqrt{2} N_{T} \sqrt{\frac{N_{T}}{N_{s}}}\right) \sqrt{\log N_{\tau} N_{f} N_{R} N_{T}}\right) \\
& \geq 1-4\left(N_{\tau} N_{f} N_{R} N_{T}\right)^{-2}-4 N_{T}\left(N_{\tau} N_{f} N_{R} N_{T}\right)^{-2}
\end{aligned}
$$

combined with (44),

$$
\begin{aligned}
\mathbb{P}\left(\left|\left\langle\mathbf{A}_{\tau, f, \beta}, \mathbf{A}_{\tau, f^{\prime}, \beta^{\prime}}\right\rangle\right|\right. & \left.\leq 8\left(\sqrt{N_{R} N_{T}}+N_{T} \sqrt{\frac{N_{R} N_{T}}{N_{s}}}\right) \log N_{\tau} N_{f} N_{R} N_{T}\right) \\
\geq & 1-8\left(N_{\tau} N_{f} N_{R} N_{T}\right)^{-2}-4 N_{T}\left(N_{\tau} N_{f} N_{R} N_{T}\right)^{-2} .
\end{aligned}
$$

After taking the union bound over $\left(N_{R} N_{T}\right)^{2}$ different possibilities associated with $\beta, \beta^{\prime}$, we will have that

$$
\begin{array}{r}
\mathbb{P}\left(\max \left|\left\langle\mathbf{A}_{\tau, f, \beta}, \mathbf{A}_{\tau, f^{\prime}, \beta^{\prime}}\right\rangle\right| \leq 8\left(\sqrt{N_{R} N_{T}}+N_{T} \sqrt{\frac{N_{R} N_{T}}{N_{s}}}\right) \log N_{\tau} N_{f} N_{R} N_{T}\right) \\
\geq 1-8 N_{\tau}^{-2} N_{f}^{-2}-4 N_{T} N_{\tau}^{-2} N_{f}^{-2} .
\end{array}
$$

A little algebra, using (20) and (21), shows that

$$
8\left(\sqrt{N_{R} N_{T}}+N_{T} \sqrt{\frac{N_{R} N_{T}}{N_{s}}}\right) \leq 16 N_{R}
$$

therefore

$$
\begin{array}{r}
\mathbb{P}\left(\max \left|\left\langle\mathbf{A}_{\tau, f, \beta}, \mathbf{A}_{\tau, f, \beta^{\prime}}\right\rangle\right| \leq 16 N_{R} \log N_{\tau} N_{f} N_{R} N_{T}\right) \\
\geq 1-8 N_{\tau}^{-2} N_{f}^{-2}-4 N_{T} N_{\tau}^{-2} N_{f}^{-2} .
\end{array}
$$

Case (ii) $\beta \neq \beta^{\prime},(\tau, f) \neq(0,0)$ :

For the same reason, here holds (44).

Define $C=\mathbf{S}_{\tau, f}^{*} \mathbf{S}$, from the properties of $\mathbf{k}_{j}$, we have that

$$
\left|c_{k j}\right|=\left|\left\langle\mathbf{M}_{f} \mathbf{T}_{\tau} \mathbf{k}_{k}, \mathbf{k}_{j}\right\rangle\right| \leq \frac{1}{\sqrt{N_{s}}} \text { for } k \neq j
$$

and there exists $j_{0}$ such that $\left|c_{j_{0} j_{0}}\right|=1$ and $c_{j j}=0$ for $j \neq j_{0}$. Then

$$
\left|\left\langle\mathbf{S}_{\tau, f}^{*} \mathbf{S a}_{T}(\beta), \mathbf{a}_{T}\left(\beta^{\prime}\right)\right\rangle\right| \leq 1+\left|\left\langle C^{\prime} \mathbf{a}_{T}(\beta), \mathbf{a}_{T}\left(\beta^{\prime}\right)\right\rangle\right|
$$

where $C^{\prime}$ is a zero-diagonal matrix which coincides $C$ at off-diagonal entries. Certainly $C^{\prime}$ satisfies the condition for (28) to hold. Choosing $t=4 \frac{\sqrt{N_{T}}}{\sqrt{N_{s}}} \sqrt{\log N_{\tau} N_{f} N_{R} N_{T}}$ in (28) of Lemma 5.17yields

$$
\begin{array}{r}
\mathbb{P}\left(\left|\left\langle\mathbf{S}_{\tau, f}^{*} \mathbf{S a}_{T}(\beta), \mathbf{a}_{T}\left(\beta^{\prime}\right)\right\rangle\right| \leq 1+4\right. \\
\left.\frac{N_{T} \sqrt{N_{T}}}{\sqrt{N_{s}}} \sqrt{\log N_{\tau} N_{f} N_{R} N_{T}} \mid\right) \\
\geq 1-4 N_{T}\left(N_{\tau} N_{f} N_{R} N_{T}\right)^{-4}
\end{array}
$$


from the assumption that $32 N_{T}^{3} \log N_{\tau} N_{f} N_{R} N_{T} \leq N_{s}$, together with (44), we will get

$$
\begin{array}{r}
\mathbb{P}\left(\left|\left\langle\mathbf{A}_{\tau, f, \beta}, \mathbf{A}_{\tau^{\prime}, f^{\prime}, \beta^{\prime}}\right\rangle\right| \leq 4 \sqrt{2} \sqrt{N_{R}} \sqrt{\log N_{\tau} N_{f} N_{R} N_{T}}\right) \\
\geq 1-4\left(N_{\tau} N_{f} N_{R} N_{T}\right)^{-2}-4 N_{T}\left(N_{\tau} N_{f} N_{R} N_{T}\right)^{-4} .
\end{array}
$$

By (21), we deduce $\log N_{\tau} N_{f} N_{R} N_{T} \leq N_{R}$. Therefore

$\mathbb{P}\left(\left|\left\langle\mathbf{A}_{\tau, f, \beta}, \mathbf{A}_{\tau^{\prime}, f^{\prime}, \beta^{\prime}}\right\rangle\right| \leq 4 \sqrt{2} N_{R}\right) \geq 1-4\left(N_{\tau} N_{f} N_{R} N_{T}\right)^{-2}-4 N_{T}\left(N_{\tau} N_{f} N_{R} N_{T}\right)^{-4}$.

We apply the union bound over $N_{\tau} N_{f} N_{R}^{2} N_{T}^{2}$ possibilities and arrive at

$$
\mathbb{P}\left(\max \left|\left\langle\mathbf{A}_{\tau, f, \beta}, \mathbf{A}_{\tau^{\prime}, f^{\prime}, \beta^{\prime}}\right\rangle\right| \leq 4 \sqrt{2} N_{R}\right) \geq 1-4\left(N_{\tau} N_{f}\right)^{-1}-4 N_{\tau}^{-3} N_{f}^{-3} N_{R}^{-2} N_{T}^{-1} .
$$

Case (iii) $\beta=\beta^{\prime},(\tau, f) \neq(0,0)$ :

Note that the matrix $C=\mathbf{S}_{\tau, f}^{*} \mathbf{S}$ has exactly the same properties as in Case (ii) above. Following the same argument as we show (46) and applying (29) of Lemma 5.1 combined with the assumption as in (20) gives us that

$$
\begin{array}{r}
\mathbb{P}\left(\left|\left\langle\mathbf{S}_{\tau, f}^{*} \mathbf{S a}_{T}(\beta), \mathbf{a}_{T}(\beta)\right\rangle\right| \leq 1+4 \sqrt{2} \frac{N_{T} \sqrt{N_{T}}}{\sqrt{N_{s}}} \sqrt{\log N_{\tau} N_{f} N_{R} N_{T}}\right) \\
\geq 1-8 N_{T}\left(N_{\tau} N_{f} N_{R} N_{T}\right)^{-4}
\end{array}
$$

which implies that

$$
\mathbb{P}\left(\left|\left\langle\mathbf{A}_{\tau, f, \beta}, \mathbf{A}_{\tau^{\prime}, f^{\prime}, \beta^{\prime}}\right\rangle\right| \leq 2 N_{R}\right) \geq 1-8 N_{T}^{-3}\left(N_{\tau} N_{f} N_{R}\right)^{-4},
$$

We apply the union bound over the $N_{\tau} N_{f} N_{R} N_{T}$ possibilities associated with $\tau$, $f$ and $\beta$

$$
\mathbb{P}\left(\max \left|\left\langle\mathbf{A}_{\tau, f, \beta}, \mathbf{A}_{\tau^{\prime}, f^{\prime}, \beta}\right\rangle\right| \leq 2 N_{R}\right) \geq 1-8 N_{T}^{-2}\left(N_{\tau} N_{f} N_{R}\right)^{-3} .
$$

(45), (47) and (48) will give the conclusion.

5.4. Regarding the matrix with unit-norm columns. In order to apply Theorem 1.3 in [5], we need to normalize the columns of $\mathbf{A}$. We first have the following result which shows the lower and upper bounds of the norm of columns of $\mathbf{A}$.

Lemma 5.4. Let $\mathbf{A}$ be defined as in Theorem 4.1 satisfying (20), then

$$
\mathbb{P}\left(\frac{1}{3} N_{R} N_{T} \leq \min \left\|\mathbf{A}_{\tau, f, \beta}\right\|_{2}^{2} \leq \max \left\|\mathbf{A}_{\tau, f, \beta}\right\|_{2}^{2} \leq \frac{5}{3} N_{R} N_{T}\right) \geq 1-8 N_{\tau}^{-2} N_{R}^{-1} .
$$

Proof. Recall that

$$
\begin{aligned}
\left\|\mathbf{A}_{\tau, f, \beta}\right\|_{2}^{2}=\left\|\mathbf{a}_{R}(\beta)\right\|_{2}^{2}\left\|\mathbf{S}_{\tau, f} \mathbf{a}_{T}(\beta)\right\|_{2}^{2} & =N_{R}\left\langle\mathbf{S}_{\tau, f}^{*} \mathbf{S}_{\tau, f} \mathbf{a}_{T}(\beta), \mathbf{a}_{T}(\beta)\right\rangle \\
& =N_{R}\left\langle\mathbf{S}^{*} \mathbf{S a}_{T}(\beta), \mathbf{a}_{T}(\beta)\right\rangle .
\end{aligned}
$$


Setting $t=2 \sqrt{\frac{N_{T}}{N_{s}}} \sqrt{\log N_{\tau} N_{R} N_{T}}$ in (31) of Lemma 5.1 yields

$$
\begin{gathered}
\mathbb{P}\left(N_{T}\left(1-4 \sqrt{\frac{N_{T}}{N_{s}}} \sqrt{\log N_{\tau} N_{R} N_{T}}\right) \leq\left|\left\langle\mathbf{S}^{*} \mathbf{S a}_{T}(\beta), \mathbf{a}_{T}(\beta)\right\rangle\right| \leq\right. \\
\left.N_{T}\left(1+4 \sqrt{\frac{N_{T}}{N_{s}}} \sqrt{\log N_{\tau} N_{R} N_{T}}\right)\right) \geq 1-8 N_{T}\left(N_{\tau} N_{R} N_{T}\right)^{-2} .
\end{gathered}
$$

An easy calculation from (20) leads

which indeed implies

$$
4 \sqrt{\frac{N_{T}}{N_{s}}} \sqrt{\log N_{\tau} N_{R} N_{T}} \leq \frac{2}{3}
$$

$$
\mathbb{P}\left(\frac{1}{3} N_{T} \leq\left|\left\langle\mathbf{S}^{*} \mathbf{S a}_{T}(\beta), \mathbf{a}_{T}(\beta)\right\rangle\right| \leq \frac{5}{3} N_{T}\right) \geq 1-8 N_{T}\left(N_{\tau} N_{R} N_{T}\right)^{-2} .
$$

Since the above probability does not depend on $\tau$ or $f$, we take all $N_{R} N_{T}$ possibilities of $\beta$ and conclude the proof of the lemma.

Corollary 5.5. Suppose $\tilde{\mathbf{A}}=\mathbf{A D}^{-1}$ where $\mathbf{D}$ is the $N_{\tau} N_{f} N_{\beta} \times N_{\tau} N_{f} N_{\beta}$ diagonal matrix defined by $\mathbf{D}_{(\tau, f, \beta),(\tau, f, \beta)}=\left\|\mathbf{A}_{\tau, f, \beta}\right\|_{2}$, or in other words $\tilde{\mathbf{A}}$ is the matrix with unit-norm columns from $\mathbf{A}$. Then

$$
\mathbb{P}\left(\|\tilde{\mathbf{A}}\|_{o p}^{2} \leq 6 N_{f} N_{R} N_{T}\right) \geq 1-16 N_{\tau}^{-2} N_{R}^{-1}
$$

and

$$
\mathbb{P}\left(\mu(\tilde{\mathbf{A}}) \leq 48 \frac{\log N_{\tau} N_{f} N_{R} N_{T}}{N_{T}}\right) \geq 1-p_{3}
$$

where

$$
\begin{aligned}
p_{3}=8 N_{\tau}^{-2} N_{R}^{-1} & +8 N_{\tau}^{-2} N_{f}^{-2}+4 N_{T} N_{\tau}^{-2} N_{f}^{-2}+4\left(N_{\tau} N_{f}\right)^{-1} \\
& +4 N_{\tau}^{-3} N_{f}^{-3} N_{R}^{-2} N_{T}^{-1}+8 N_{T}^{-2}\left(N_{\tau} N_{f} N_{R}\right)^{-3} .
\end{aligned}
$$

Proof. This corollary is a direct consequence of Lemma 5.2 Lemma 5.3 and Lemma 5.4 .

\subsection{Assembling the proof of Theorem 4.1.}

Proof. (of Theorem 4.1) Recall that we are trying to use Theorem 1.3 in [5] to prove Theorem 4.1. We thus need to verify that all assumptions of that theorem are satisfied.

We first point out that the assumptions of Theorem 4.1 imply that the conditions of Lemma 5.2 and Lemma 5.3 are fulfilled.

Note that solution $\tilde{\mathbf{x}}$ of (19) and the solution $\tilde{\mathbf{z}}$ of the following lasso problem

$$
\min _{\mathbf{z}} \frac{1}{2}\left\|\mathbf{A D}^{-1} \mathbf{z}-\mathbf{y}\right\|_{2}^{2}+\lambda\|\mathbf{z}\|_{1}, \quad \text { with } \lambda=2 \sigma \sqrt{2 \log \left(N_{\tau} N_{R} N_{T}\right)},
$$

are related by $\tilde{\mathbf{x}}=\mathbf{D}^{-1} \tilde{\mathbf{z}}$.

We will first establish the claims in Theorem 4.1 for the system $\tilde{\mathbf{A}} \mathbf{z}=\mathbf{y}$ where $\tilde{\mathbf{A}}=\mathbf{A D}^{-1}, \mathbf{z}=\mathbf{D} \mathbf{x}$.

First, the assumption (23) and the fact that $\mathbf{z}=\mathbf{D x}$ imply that $\left|z_{k}\right| \geq \frac{8 \sqrt{3} \min \left\|\mathbf{A}_{\tau, f, \beta}\right\|_{2}}{\sqrt{N_{R} N_{T}}} \sigma \sqrt{2 \log \left(N_{\tau} N_{f} N_{\beta}\right)} \geq 8 \sigma \sqrt{2 \log \left(N_{\tau} N_{f} N_{\beta}\right)}, \quad$ for $k \in S$, 
with probability at least $1-8 N_{\tau}^{-2} N_{R}^{-1}$, thus establishing the first condition of Theorem 1.3 in $[5$.

Using the assumptions in Theorem 4.1, and the coherence bound (52) we compute

$$
\mu(\tilde{\mathbf{A}}) \leq 48 \frac{\log N_{\tau} N_{f} N_{R} N_{T}}{N_{T}} \leq \frac{48}{\log N_{\tau} N_{f} N_{\beta}},
$$

which holds with probability as in (52), and thus the coherence property (1.5) in [5] is fulfilled.

Furthermore, using (51) we see that condition (22) implies

$$
S \leq \frac{c_{0} N_{\tau}}{\log \left(N_{\tau} N_{f} N_{\beta}\right)} \leq \frac{6 c_{0} N_{\tau} N_{f} N_{\beta}}{\|\tilde{\mathbf{A}}\|_{\mathrm{op}}^{2} \log \left(N_{\tau} N_{f} N_{\beta}\right)}
$$

with probability at least $1-16 N_{\tau}^{-2} N_{R}^{-1}$. Thus the sparsity assumption of Theorem 1.3 in [5] is also fulfilled and we obtain that

$$
\operatorname{supp}(\tilde{\mathbf{z}})=\operatorname{supp}(\mathbf{z})
$$

with probability at least $1-p_{1}$. We note that the relation $\operatorname{supp}(\tilde{\mathbf{x}})=\operatorname{supp}(\mathbf{x})$ holds with the same probabiltity as the relation $\operatorname{supp}(\tilde{\mathbf{z}})=\operatorname{supp}(\mathbf{z})$, since $\operatorname{supp}(\mathbf{z})=$ $\operatorname{supp}(\mathbf{x})$ and multiplication by an invertible diagonal matrix does not change the support of a vector. This establishes (24) with the corresponding probability.

Once we have recovered the support of $\mathbf{x}$, call it $I$, we can solve for the coefficients of $\mathbf{x}$ by solving the standard least squares problem min $\left\|\mathbf{A}_{I} \mathbf{x}_{I}-\mathbf{y}\right\|_{2}$, where $\mathbf{A}_{I}$ is tbe submatrix of $\mathbf{A}$ whose columns correspond to the support set $I$, and similarly for $\mathbf{x}_{I}$. Note that the proof of Theorem 3.2 in [5] yields as side result that with high probability the eigenvalues of any submatrix $\mathbf{A}_{I}^{*} \mathbf{A}_{I}$ with $|I| \leq S$ are contained in the interval $[1 / 2,3 / 2]$, which of course implies that $\kappa\left(\mathbf{A}_{I}\right) \leq \sqrt{3}$. By substituting this bound into the standard error bound, (5.8.11) in [19], we have that

$$
\frac{\|\tilde{\mathbf{z}}-\mathbf{z}\|_{2}}{\|\mathbf{z}\|_{2}} \leq \frac{\sigma \sqrt{3 N_{R} N_{s}}}{\|\mathbf{y}\|_{2}}
$$

which holds with probability at least

$$
\left(1-p_{1}\right)\left(1-p_{2}\right)
$$

Using the fact that $\tilde{\mathbf{z}}=\mathbf{D} \tilde{\mathbf{x}}$, we compute

$$
\frac{1}{\kappa(\mathbf{D})} \frac{\|\tilde{\mathbf{x}}-\mathbf{x}\|_{2}}{\|\mathbf{x}\|_{2}} \leq \frac{\|\mathbf{D}(\tilde{\mathbf{x}}-\mathbf{x})\|_{2}}{\|\mathbf{D} \mathbf{x}\|_{2}}=\frac{\|\tilde{\mathbf{z}}-\mathbf{z}\|_{2}}{\|\mathbf{z}\|_{2}}
$$

or, equivalently,

$$
\frac{\|\tilde{\mathbf{x}}-\mathbf{x}\|_{2}}{\|\mathbf{x}\|_{2}} \leq \kappa(\mathbf{D}) \frac{\|\tilde{\mathbf{z}}-\mathbf{z}\|_{2}}{\|\mathbf{z}\|_{2}}
$$

The bound (26) follows now from combining (58), (60) with the fact that $\kappa(\mathbf{D}) \leq 5$ (from Lemma 5.4). 


\section{Extension of the main Result: Beyond Kerdock Codes}

In this section, we present a modified version of Theorem 4.1 that applies to waveforms that satisfy slightly more restrictive incoherence conditions. As such, Theorem 6.1 below does not hold for Kerdock waveforms, but the advantage compared to Theorem 4.1 is that the result also applies to radar systems with only one transmit antenna.

Theorem 6.1. Consider $\mathbf{y}=\mathbf{A x}+\mathbf{w}$, where $\mathbf{A}$ is defined as in (10) and $\mathbf{w}_{j} \in$ $\mathcal{C} N\left(0, \sigma^{2}\right)$. Suppose the transmission waveforms $\mathbf{s}_{j}$ 's satisfy the following conditions

$$
\begin{aligned}
& \left|\left\langle\mathbf{s}_{j}, \mathbf{M}_{f} \mathbf{T}_{\tau}\right\rangle \mathbf{s}_{j}\right| \leq \frac{\gamma}{\sqrt{p}} \text { for }(f, \tau) \neq(0,0), \\
& \left|\left\langle\mathbf{s}_{k}, \mathbf{M}_{f} \mathbf{T}_{\tau}\right\rangle \mathbf{s}_{j}\right| \leq \frac{\gamma}{\sqrt{p}} \text { for } k \neq j
\end{aligned}
$$

where $\gamma>0$ is a fixed constant. Assume that the positions of the transmit and receive antennas $p_{j}$ 's and $q_{j}$ 's are chosen i.i.d. uniformly on $\left[0, \frac{N_{R} N_{T}}{2}\right]$ at random. Choose the discretization stepsizes to be $\Delta_{\beta}=\frac{2}{N_{R} N_{T}}, \Delta_{\tau}=\frac{1}{2 B}, \Delta_{f}=\frac{1}{T}$ and suppose that

$$
\max \left(\gamma^{2} N_{R} N_{T}, 16 \gamma^{2} N_{T} \log ^{3} N_{\tau} N_{f} N_{\beta}\right) \leq N_{s}=N_{\tau}
$$

and also

$$
\gamma^{2} N_{T} \log ^{4} N_{\tau} N_{f} N_{\beta} \leq N_{s} N_{R}, \quad \log ^{2} N_{\tau} N_{f} N_{\beta} \leq N_{T} \leq N_{R}
$$

Then if the rest of the conditions of Theorem 4.1 hold, we have the same conclusion as in Theorem 4.1 .

Proof. The proof of this theorem is similar to the one of Theorem 4.1. The main difference will arise when estimating the coherence of the matrix $\mathbf{A}$. (45) will remain the same except with an extra $\gamma$ factor. From the conditions of the waveforms above, (47) becomes

$$
\begin{aligned}
\mathbb{P}\left(\max \left|\left\langle\mathbf{A}_{\tau, f, \beta}, \mathbf{A}_{\tau^{\prime}, f^{\prime}, \beta^{\prime}}\right\rangle\right|\right. & \left.\leq 8 \sqrt{2} \frac{\gamma N_{T} \sqrt{N_{T} N_{R}}}{\sqrt{N_{s}}} \log N_{\tau} N_{f} N_{R} N_{T}\right) \\
\geq & 1-4\left(N_{\tau} N_{f}\right)^{-1}-4 N_{\tau}^{-3} N_{f}^{-3} N_{R}^{-2} N_{T}^{-1},
\end{aligned}
$$

and we have to change (48) in the same manner.

Then from the new conditions (63) and (64), we will have a similar estimate for the coherence of the normalized matrix $\tilde{\mathbf{A}}$ as in (55).

There are several examples of signal sets that satisfy the above conditions. Perhaps the most intriguing example is the finite harmonic oscillator system (FHOS) constructed in 14. This signal set in $\mathbb{C}^{p}$ (where $p$ is a prime number) of cardinality $\mathcal{O}\left(p^{3}\right)$ satisfies (61) and (62) with $\gamma=4$. An elementary construction of the FHOS for prime number $p \geq 5$ can be found in [37. We illustrate the performance of the FHOS and its comparison to Kerodck codes in numerical simulations in the next section. 


\section{NUMERICAL SIMULATIONS}

In this section we will demonstrate the performance of our algorithms via numerical simulations. We use the Matlab Toolbox TFOCS ([2]) and choose in TFOCS Auslender and Teboulle's single-projection method to solve (19). The main computational costs per iteration of this method are the operations $\mathbf{A} x$ and $\mathbf{A}^{*} y$. One can of course make $\mathbf{A}$ explicit and do the regular matrix multiplication, but due to the special structure of $\mathbf{A}$, we make the following observation to accelerate the computation.

Recall that

$$
\mathbf{A}_{\tau, f, \beta}=\mathbf{a}_{R}(\beta) \otimes\left(\mathbf{S}_{\tau, f} \mathbf{a}_{T}(\beta)\right) .
$$

First suppose we have $x$ given and we want to compute $y=\mathbf{A} x$. Here $y$ is an $N_{R} N_{s} \times 1$ vector and $x$ is an $N_{\tau} N_{f} N_{\beta} \times 1$ vector. Instead of doing the direct matrix-vector multiplication, we divide $y$ into $N_{R}$ blocks $y_{j}$, each of which is of size $N_{s} \times 1$. Then

$$
\begin{aligned}
y_{j} & =\sum_{\tau, \beta, f} \mathbf{a}_{R}(\beta)_{j} \mathbf{S}_{\tau, f} \mathbf{a}_{T}(\beta) x(\tau, \beta, f) \\
& =\sum_{\beta} \mathbf{a}_{R}(\beta)_{j} \sum_{\tau, f} \mathbf{S}_{\tau, f} \mathbf{a}_{T}(\beta) x(\tau, \beta, f) .
\end{aligned}
$$

Since for any fixed $\beta$ and $f$, we can consider $x_{\beta, f}(\tau)=x(\tau, \beta, f)$ as an $N_{\tau} \times 1$ vector. Then, recalling that $\mathbf{S}_{\tau, f} \mathbf{a}_{T}(\beta)=\mathbf{M}_{f} \mathbf{T}_{\tau}\left(\mathbf{S a}_{T}(\beta)\right)$, an easy observation yields

$$
\begin{aligned}
y_{j} & =\sum_{\beta} \mathbf{a}_{R}(\beta)_{j} \sum_{f} \mathbf{M}_{f}\left(\sum_{\tau} \mathbf{T}_{\tau} \mathbf{S a}_{T}(\beta) x(\tau, \beta, f)\right) \\
& =\sum_{\beta} \mathbf{a}_{R}(\beta)_{j} \sum_{f} \mathbf{M}_{f}\left(\mathbf{S a}_{T}(\beta) * x_{\beta, f}\right),
\end{aligned}
$$

where the convolution can be implemented via FFT.

Now we suppose that $y$ is given and we want to compute $x=\mathbf{A}^{*} y$. Note that in this case we have that the row vector of $\mathbf{A}^{*}$ is of the form

$$
\mathbf{A}_{\tau, f, \beta}^{*}=\mathbf{a}_{R}(\beta)^{*} \otimes\left(\mathbf{S}_{\tau, f} \mathbf{a}_{T}(\beta)\right)^{*}
$$

We divide $x$ into $N_{\beta} N_{f}$ blocks $x_{\beta, f}$, each of which is of size $N_{\tau}$. We also divide $y$ into $N_{R}$ blocks $y_{j}$, each of which is of size $N_{s}$.

$$
x_{\beta, f}=\sum_{j=1}^{N_{R}} \overline{\mathbf{a}_{R}(\beta)_{j}} \mathbf{C}_{\beta, f} y_{j},
$$

where $\mathbf{C}_{\beta, f}$ is the matrix whose rows are $\left(\mathbf{S}_{\tau, f} \mathbf{a}_{T}(\beta)\right)^{*}=\left(\mathbf{M}_{f} \mathbf{S}_{\tau} \mathbf{a}_{T}(\beta)\right)^{*}$, an easy calculation leads us to

$$
x_{\beta, f}=\sum_{j=1}^{N_{R}} \overline{\mathbf{a}_{R}(\beta)_{j}} \mathbf{B}_{\beta}\left(\mathbf{M}_{f} y_{i}\right),
$$

where $\mathbf{B}_{\beta}$ is the matrix whose rows are $\left(\mathbf{S}_{\tau} \mathbf{a}_{T}(\beta)\right)^{*}$. So $\mathbf{B}_{\beta}$ is a circulant matrix and its first column $c_{\beta}$ is that $c_{\beta}(1)=\overline{\mathbf{S a}_{T}(\beta)(1)}$ and $c_{\beta}(k)=\overline{\mathbf{S a}_{T}(\beta)\left(N_{s}-k\right)}$ for $k=2, \ldots, N_{s}$. Then we have $\mathbf{B}_{\beta}\left(\mathbf{M}_{f} y_{j}\right)=c_{\beta} \otimes \mathbf{M}_{f} y_{j}$, which implies

$$
x_{\beta, f}=\sum_{j=1}^{N_{R}} \overline{\mathbf{a}_{R}(\beta)_{j}}\left(c_{\beta} \otimes \mathbf{M}_{f} y_{j}\right) \text {. }
$$


In each experiment, the locations of the transmit and receive antennas are chosen i.i.d. randomly on $\left[0, \frac{N_{R} N_{T}}{2}\right]$ and $S$ scatterers are placed randomly on the rangeazimuth-Doppler grid, i.e the vector $x$ has $S$ entries at random locations along the vector. White Gaussian noise is added to the composite data vector $\mathbf{A} x$ with variance $\sigma^{2}$ determined to produce the specified output signal-to-noise ratio. The lasso solution $\hat{x}$ is calculated with $\lambda$ as specified in Theorem 4.1. The experiment is repeated 50 times. Each experiment uses independent noise realization.

The probabilities of detection $P_{d}$ and false alarm $P_{f a}$ are computed as follows. The values of the estimated vector $\hat{x}$ corresponding to the true scatterer locations are compared to a threshold. Detection is declared whenever a value exceeds the threshold. The probability of detection is defined as the number of detections divided by the total number of scatterers $S$. Next the values of the estimated vector $\hat{x}$ corresponding to locations not containing scatterers are compared to the same threshold. A false alarm is declared whenever one of these values exceeds the threshold. The probability of false alarm is defined as the number of false alarms divided by $n-S$, where $n$ is the signal dimension. The probabilities of detection and false alarm are averaged over the 50 repetitions of the experiment.

The probabilities are computed for a range of values of the threshold to produce the so-called Receiver Operating Characteristics (ROC) [14, 28, 25] - the graph of $P_{d}$ vs. $P_{f a}$. As the threshold decreases, the probability of detection increases and so does the probability of false alarm. In practice the threshold is usually adjusted to achieve a specified probability of false alarm. We note that the probability of detection increases as the SNR increases and decreases as $S$, the number of scatterers increases.

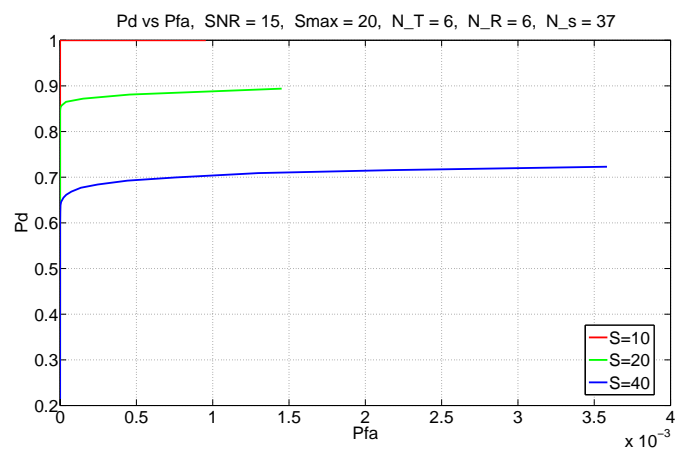

Figure 2. MIMO, Kerdock, SNR=15

We carry out two sets of simulations with different parameters to show the performance of the algorithms.

(1) The first set of simulations is done using Kerdock codes as transmission waveforms. The following parameters are used: $N_{T}=6, N_{R}=6, N_{s}=$ $37, N_{f}=37$ (hence we are using Kerdock waveforms of length 37 ); $S_{\max }=$ 20 and the actual number of targets is $S=S_{\max } / 2, S_{\max }, 2 S_{\max }$, while the SNR is chosen to be $15 \mathrm{~dB}, 20 \mathrm{~dB}$ and $25 \mathrm{~dB}$ in Figure 2, Figure 3 and 


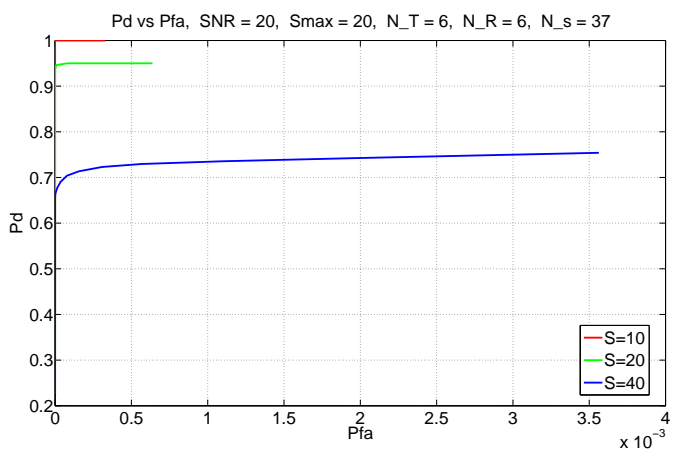

Figure 3. MIMO, Kerdock codes, $\mathrm{SNR}=20$

Figure 4 respectively. This set of simulations is aimed to demonstrate the efficiency of Kerdock codes for MIMO radar at different SNR levels.

(2) The second set of simulations is to compare the Kerdock codes and the finite harmonic oscillator system for both SIMO and MIMO. We fix the following parameters in this set of simulations: $S_{\max }=10$ and $S=S_{\max } / 2, S_{\max }, 2 S_{\max }$, while the SNR is fixed to be $15 \mathrm{~dB}$. We choose $N_{T}=1, N_{R}=8, N_{s}=$ $11, N_{f}=11$ for SIMO and $N_{T}=2, N_{R}=8, N_{s}=17, N_{f}=17$ for MIMO. As we mentioned before, Figure 5 shows that Kerdock codes are not very good choice in SIMO. But Kerdock codes are already very efficient when $N_{T}=2$ as shown in Figure 6, which also shows that the condition $\log ^{2} N_{\tau} N_{f} N_{\beta} \leq N_{T}$ in (21) is a restrictive theoretical condition and we can do much better in practice.

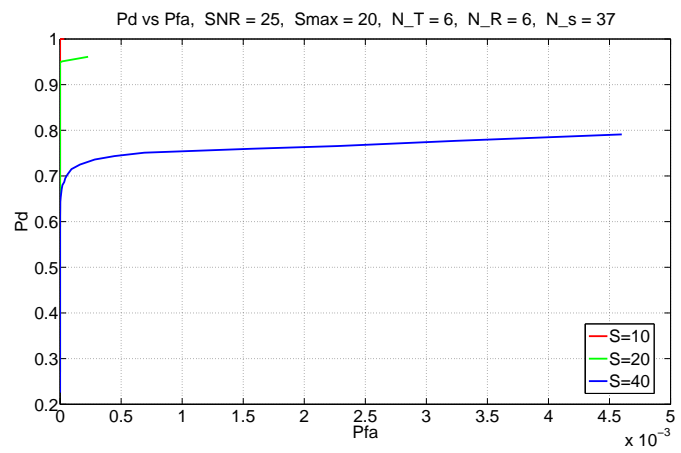

Figure 4. MIMO, Kerdock codes, SNR=25

\section{REFERENCES}

[1] W. O. Alltop. Complex sequences with low periodic correlations. IEEE Trans. on Information Theory, 26(3):350-354, 1980. 


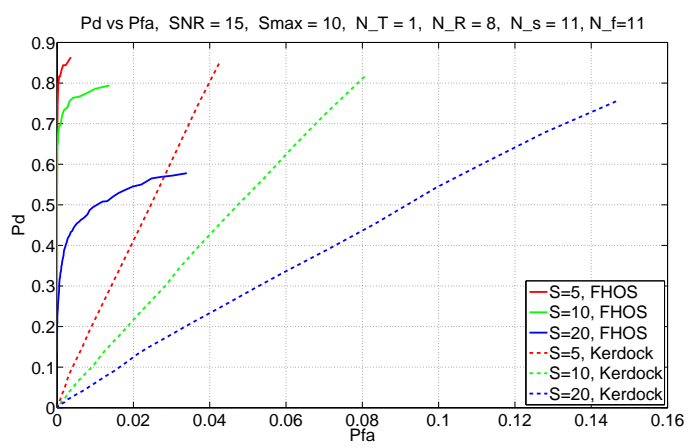

Figure 5. SIMO, Kerdock codes vs FHOS, SNR=15

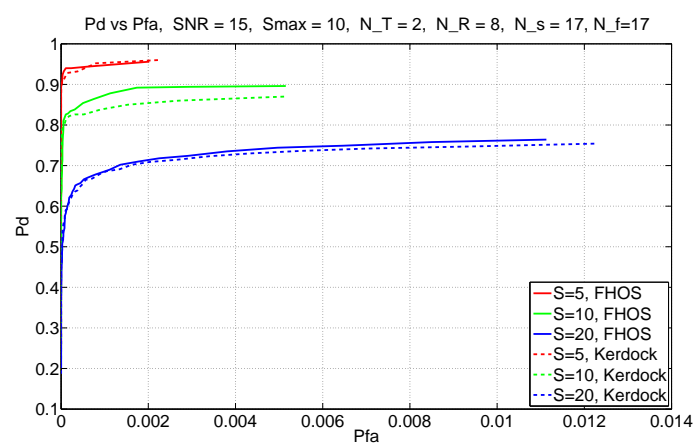

Figure 6. MIMO, Kerdock codes vs FHOS, SNR=15

[2] S. Becker, E. Candes, and M. Grant. Templates for convex cone problems with applications to sparse signal recovery. Mathematical Programming Computation, 3(3):165-218, 2011.

[3] J.J. Benedetto, R. Benedetto, and J.T. Woodworth. Optimal ambiguity functions and weil's exponential sum bound. Journal of Fourier Analysis and Applications, 18:471-487, 2012.

[4] A. R. Calderbank, P. J. Cameron, W. M. Kantor, and J. J. Seidel. $Z_{4}$-Kerdock codes, orthogonal spreads, and extremal Euclidean line-sets. Proc. London Math. Soc. (3), 75(2):436-480, 1997.

[5] E.J. Candès and Y. Plan. Near-ideal model selection by $\ell_{1}$ minimization. Annals of Statistics, $37(5 \mathrm{~A}): 2145-2177,2009$.

[6] L. Carin. On the relationship between compressive sensing and random sensor arrays. IEEE Antennas and Propagation Magazine, 51(5):72-81, 2009.

[7] V. Cevher, P. T. Boufounos, R. G. Baraniuk, A. C. Gilbert, and M. J. Strauss. Near-optimal bayesian localization via incoherence and sparsity. In Proc. of the 2009 International Conference on Information Processing in Sensor Networks (IPSN), pages 205-216, April 13-16 2009.

[8] Y. Chi, L.L. Scharf, A. Pezeshki, and A.R. Calderbank. Sensitivity to basis mismatch in compressed sensing. IEEE Trans. Signal Processing, 59(5):2182-2195, 2011.

[9] J.P. Costas. A study of a class of detection waveforms having nearly ideal range-doppler ambiguity properties. Proceedings of the IEEE, 72(8):996-1009, 1984.

[10] A. Fannjiang and W. Liao. Coherence pattern-guided compressive sensing with unresolved grids. SIAM J. Imaging Sci., 5:179-202, 2012.

[11] A.J. Fenn, D.H. Temme, W.P. Delaney, and W.E. Courtney. The development of phased-array radar technology. Lincoln Lab. J., 12(2):321-340, 2000. 
[12] B. Friedlander. Adaptive Signal Design for MIMO Radar. In J. Li and P. Stoica, editors, MIMO Radar Signal Processing, chapter 5. John Wiley \& Sons, 2009.

[13] S.W. Golomb and G. Gong. Signal Design for Good Correlation for Wireless Communication, Cryptography and Radar. Cambridge University Press, 2005.

[14] S. Gurevich, R. Hadani, and N. Sochen. The finite harmonic oscillator and its applications to sequences, communication and radar. IEEE Trans. Inf. Theory, 54(9):4239-4253, 2008.

[15] A.M. Haimovich, R.S. Blum, and L.J. Cimini. MIMO radar with widely separated antennas. IEEE Signal Processing Magazine, pages 116-129, 2008.

[16] R. Heath, T. Strohmer, and A. Paulraj. On quasi-orthogonal signatures for CDMA systems. IEEE Trans. Info. Theory, 52(3):1217-1226, 2006.

[17] M. Herman and T. Strohmer. High-resolution radar via compressed sensing. IEEE Trans. on Signal Processing, 57(6):2275-2284, 2009.

[18] M. Herman and T. Strohmer. General deviants: an analysis of perturbations in compressed sensing. IEEE Journal of Selected Topics in Signal Processing: Special Issue on Compressive Sensing, 4(2):342-349, 2010.

[19] R.A. Horn and C.R. Johnson. Matrix analysis. Cambridge University Press, Cambridge, 1990. Corrected reprint of the 1985 original.

[20] S. D. Howard, A. R. Calderbank, and W. Moran. The finite Heisenberg-Weyl groups in radar and communications. EURASIP J. Appl. Signal Process., 2006:1-12, 2006.

[21] M. Hügel, H. Rauhut, and T. Strohmer. Remote sensing via $\ell_{1}$-minimization. 2012. Preprint.

[22] T. Inoue and R. W. Heath. Kerdock codes for limited feedback mimo systems. Proc. of IEEE Int. Conf. on Acoustics, Speech and Signal Processing, pages 3113-3116, 2008.

[23] A. Kerdock. Studies of low-rate binary codes (ph.d. thesis abstr.). IEEE Trans. Inf. Theory, 18(2):316-316, 1972.

[24] H. König. Isometric embeddings of euclidean spaces into finite-dimensional $\ell_{p}$-spaces. Banach Center Publications, 34:79-87, 1995.

[25] V.I. Levenstein. Bounds on the maximal cardinality of a code with bounded modulus of the inner product. Soviet Math. Dokl., 25:526-531, 1982.

[26] J. Li and P. Stoica. MIMO Radar with Colocated Antennas: Review of Some Recent Work. IEEE Signal Processing Magazine, 24(5):106-114, 2007.

[27] Y. Lo. A mathematical theory of antenna arrays with randomly spaced element. IEEE Trans. Antennas and Propagation, 12(3):257-268, 1964.

[28] Y. Lo. A probalistic approach to the problem of large antenna arrays. Journal of Research of the National Bureau of Standards, 68D(5):1011-1019, 1964.

[29] A. Pezeshki, A.R. Calderbank, W. Moran, and S.D. Howard. Doppler resilient Golay complementary waveforms. IEEE Transactions on Information Theory, 54(9):4254-4266, 2008.

[30] G. E. Pfander, H. Rauhut, and J. Tanner. Identification of matrices having a sparse representation. IEEE Trans. Signal Processing, 56(11):5376-5388, 2008.

[31] L. C. Potter, E. Ertin, J. T. Parker, and M. Cetin. Sparsity and compressed sensing in radar imaging. Proceedings of the IEEE, 98(6):1006-1020, 2010.

[32] A. W. Rihaczek. High-Resolution Radar. Artech House, Boston, 1996. (originally published: McGraw-Hill, NY, 1969).

[33] T. Strohmer and B. Friedlander. Analysis of sparse MIMO radar. 2012. Preprint.

[34] T. Strohmer and R. Heath. Grassmannian frames with applications to coding and communications. Applied and Computational Harmonic Analysis, 14(3):257-275, 2003.

[35] G. Tang, B.N. Bhaskar, P. Shah, and B. Recht. Compressed sensing off the grid. Preprint, [arvix:120\%.6053], 2012.

[36] R. Tibshirani. Regression shrinkage and selection via the lasso. J. Roy. Statist. Soc. Ser. B, 58(1):267-288, 1996.

[37] Z. Wang and G. Gong. New sequences design from Weil representation with low twodimensional correlation in both time and phase shifts. IEEE Trans. Inf. Theory, 57(7):46004611, 2011.

[38] G. Welti. Quaternary codes for pulsed radar. IEEE Trans. Inform. Theory, 6(3):400-408, 1960.

[39] W.K. Wootters and B.D. Fields. Optimal state-determination by mutually unbiased measurements. Annals of Physics, 191(2):363-381, 1989. 
(Thomas Strohmer) Department of Mathematics, University of California, Davis, CA 95616

E-mail address: strohmer@math.ucdavis.edu

(Haichao Wang) Department of Mathematics, University of California, Davis, CA 95616

E-mail address: hchwang@ucdavis.edu 